\title{
Novel electroluminescence technique to analyze mixed reverse breakdown phenomena in silicon diodes
}

\author{
Monuko du Plessis ${ }^{\mathrm{a}, *}$, Pieter Rademeyer ${ }^{\mathrm{b}}$ \\ ${ }^{\text {a }}$ Carl and Emily Fuchs Institute for Microelectronics, University of Pretoria, Pretoria 0002, South Africa \\ ${ }^{\mathrm{b}}$ InSiAva (Pty) Ltd., P.O. Box 14679, Hatfield 0028, Pretoria, South Africa
}

\section{A R T I C L E I N F O}

\section{Article history:}

Received 9 July 2009

Received in revised form 3 December 2009 Accepted 5 January 2010

Available online $\mathrm{xxxx}$

The review of this paper was arranged by A. Zaslavsky

\section{Keywords:}

Avalanche breakdown

Electroluminescence

Tunneling

\begin{abstract}
A B S T R A C T
In this paper a novel technique to analyze the low-voltage breakdown regime of silicon diodes is presented. It is shown that the field emission tunnel current component of the reverse current does not cause energy transitions of carriers, and therefore will not emit photons. All photons being emitted from the pn junction are due to avalanche electroluminescence as a result of hot carrier energy relaxation processes. Measuring the light intensity output as a function of reverse current, the two current components (field emission and impact ionization) can be extracted as a function of reverse voltage. The experimental results were verified using the differential dynamic impedance method, as well as fitting a theoretical model to the extracted tunnel current. The temperature coefficient of current also indicated the transition from tunneling to avalanche.
\end{abstract}

(c) 2010 Published by Elsevier Ltd.

\section{Introduction}

The reverse breakdown characteristics of silicon pn junctions have been investigated intensively over many years [1]. However, even today, the low-voltage breakdown phenomenon of mixed field emission and impact ionization has not been fully understood, or the low-voltage reverse breakdown current analyzed in terms of the two different physical mechanisms [2]. One of the very first publications on mixed breakdown regime was published in 1957 [3]. The reverse current properties and the carrier multiplication characteristics of low-voltage reverse breakdown diodes were investigated by the photo-induced injection of carriers into the junction. The multiplication factor $M$ was defined as the ratio of the photocurrent at a given voltage to its constant value at small reverse bias voltages. The multiplication factor $M$ was determined to be less than 2 for reverse bias voltages up to $3.6 \mathrm{~V}$ [3]. The break over voltage of $3.6 \mathrm{~V}$ was described as the average value of reverse voltage required for field emission carriers to acquire sufficient energy to produce impact ionization electron-hole pairs before leaving the junction, and the onset of an increasing current represents multiplication of field emission carriers.

The field emission to avalanche transition region was also determined by measuring the differential dynamic impedance of the reverse-biased diodes [4]. It was concluded that in the transition region the current is primarily caused by multiplied zener

\footnotetext{
* Corresponding author. Tel.: +27 12420 2952; fax: +27 123625115 .

E-mail address: monuko@up.ac.za (M.du Plessis).
}

current. The starting point for multiplication in the transitional breakdown voltage region was determined as a reverse bias voltage ranging between $3.3 \mathrm{~V}$ and $4.5 \mathrm{~V}$. A more detailed analysis of breakdown in silicon [5] also established that the hardness in the reverse characteristics at a voltage slightly in excess of $3 \mathrm{~V}$ is caused by the avalanche multiplication of field emission carriers. Since the field emission carriers travel on average a shorter distance through the depletion region than the thermally generated carriers (dominated in silicon by the recombination-generation current of carriers in the depletion region), the multiplication gains for the two current components will be different. In a low-voltage device (breakdown voltage less than $6 \mathrm{~V}$ ) the current in the narrow junction devices is almost entirely caused by internal field emission [5].

A significant contribution was made in 1976 [6] to distinguish the tunneling current from the impact ionization multiplied current, using noise as diagnostic tool. If the randomly emitted tunneling electrons undergo multiplication due to impact ionization, the randomness of the multiplication process generates additional noise, and the noise ratio becomes greater than unity. The results show that the departure of the reverse current from the tunneling relation is due to the impact ionization multiplication of the tunneling current. The simulation of low-voltage breakdown devices [7] resulted in the principal conclusion that the transition from tunneling to impact ionization occurs when the energy of the tunneling electrons exceeds the electron threshold energy of ionization. A transition voltage of approximately $4 \mathrm{~V}$ was observed [6] in the noise characteristics, and attributed to transitions from a 
maximum of two to a maximum of three ionizing collisions per electron transit across the diode space charge layer.

Recently, photo-induced avalanche multiplication studies to measure the avalanche multiplication factors, even in the presence of large tunneling currents, were performed $[8,9]$. The two experimental studies were similar, but more advanced, than the original work reported in 1957 [1]. The data in [8] is for diode depletion region widths down to $100 \mathrm{~nm}$, while [9] extends the measurements to diode depletion region widths as small as $30 \mathrm{~nm}$. Both reported avalanche gains very similar to the results previously published [3].

In this paper we present a new and novel technique to distinguish between the field emission and avalanche currents in lowvoltage silicon diodes, using the light emission properties from these devices $[10,11]$. It was noted very early on that very faint light is emitted from low-voltage field emission diodes compared to avalanche diodes, almost a factor 100 smaller in intensity [3]. It was also reported recently that tunneling currents do not produce hot carriers and the resultant light emission, and can be viewed as a parasitic current, which decreases the internal quantum efficiency of silicon light emitting devices [12].

\section{Low-voltage silicon devices}

The $\mathrm{n}^{+} \mathrm{p}^{+}$silicon LED device was manufactured at Austriamicrosystems (AMS), Austria, in a $0.8 \mu \mathrm{m}$ BiCMOS process through the Europractice program. The structure of the diode is shown in Fig. 1, and the reverse current vs. reverse voltage characteristic as measured with a HP4155B semiconductor parameter analyzer is shown in Fig. 2. In this figure the deviation from the tunnel current behavior can be observed as an additional increase in reverse current at a voltage just below $4 \mathrm{~V}$.

\section{Light emission}

The visible light emission from the diode was in the form of a $30 \mu \mathrm{m}$ long line segment where the $\mathrm{n}^{+}$and $\mathrm{p}^{+}$regions overlap. The linear segment light pattern is presented in Fig. 3. Optical light intensity measurements were performed using a Hamamatsu R955 photomultiplier tube. A discrepancy was observed in the light

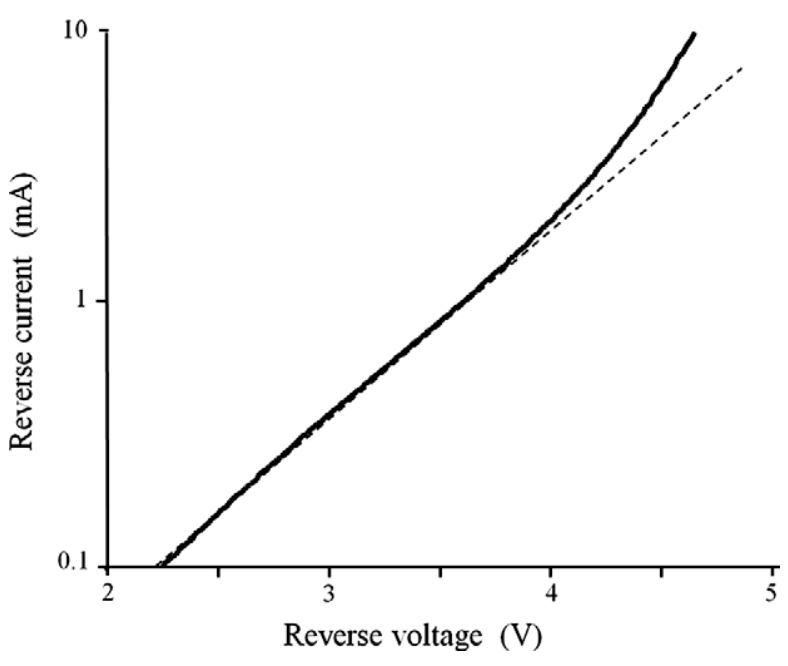

Fig. 2. Reverse current versus voltage characteristic of the $\mathrm{n}^{+} \mathrm{p}^{+}$diode.

emission versus reverse current characteristics of the low-voltage silicon $\mathrm{n}^{+} \mathrm{p}^{+}$diodes $[10,11]$. Previously, diodes operating in the avalanche mode of operation with higher breakdown voltages, exhibited a linear optical output power versus reverse current relationship, however, the low-voltage devices exhibit a non-linear behavior at low currents levels, as seen in Fig. 4 [11].

The non-linear effect is attributed to the co-existence of a field emission (or tunneling current) and an avalanche current component as discussed in the introduction above. The tunnel current does not contribute to the optical power generation process, but only the avalanche component of current. The tunnel current can thus be extracted from the optical power versus reverse current transfer characteristics.

The assumption that the tunnel current does not contribute to optical power output can be attributed to the effect that the tunnel current carriers do not undergo band to band energy shifts, but only indirect electron tunneling takes place from the valence band on the p-side to the to available empty ionized sites in the conduction band on the n-side, with phonon and momentum energy dissipation in the lattice $[13,14]$.

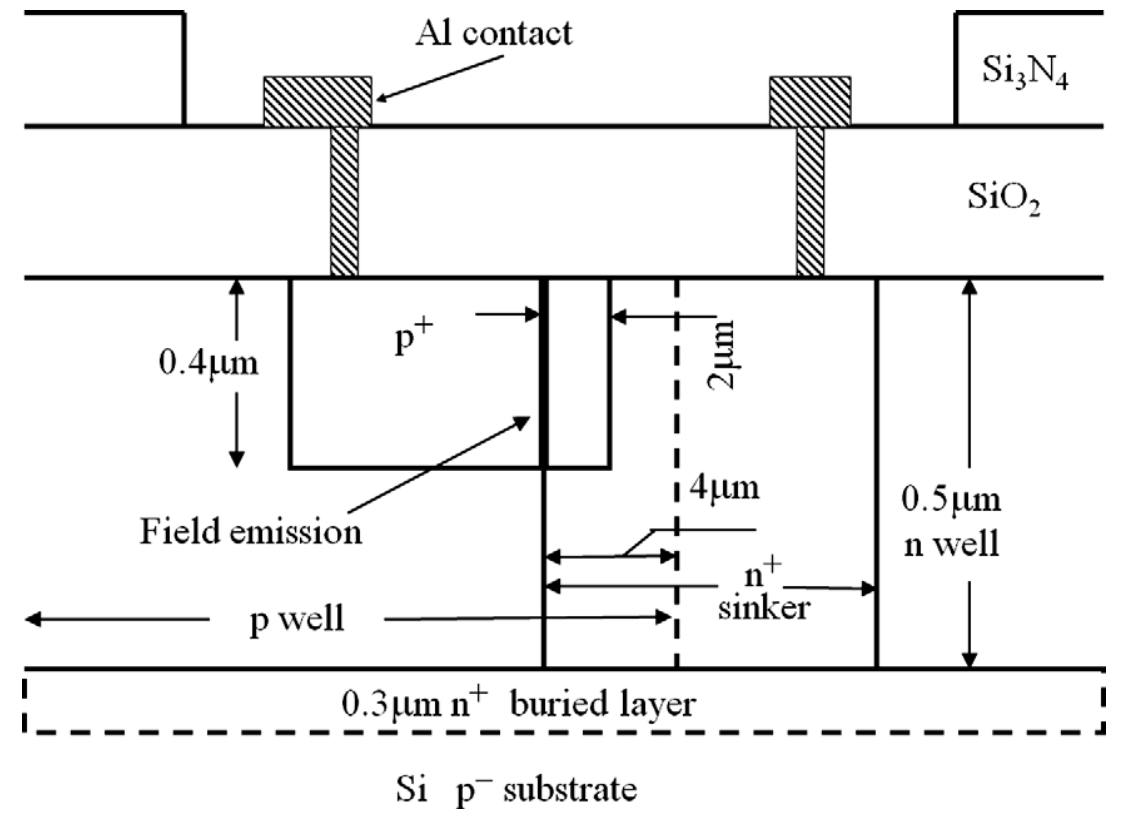

Fig. 1. Structure of the low-voltage $n^{+} p^{+}$diode in a $0.8 \mu \mathrm{m}$ BiCMOS process. 


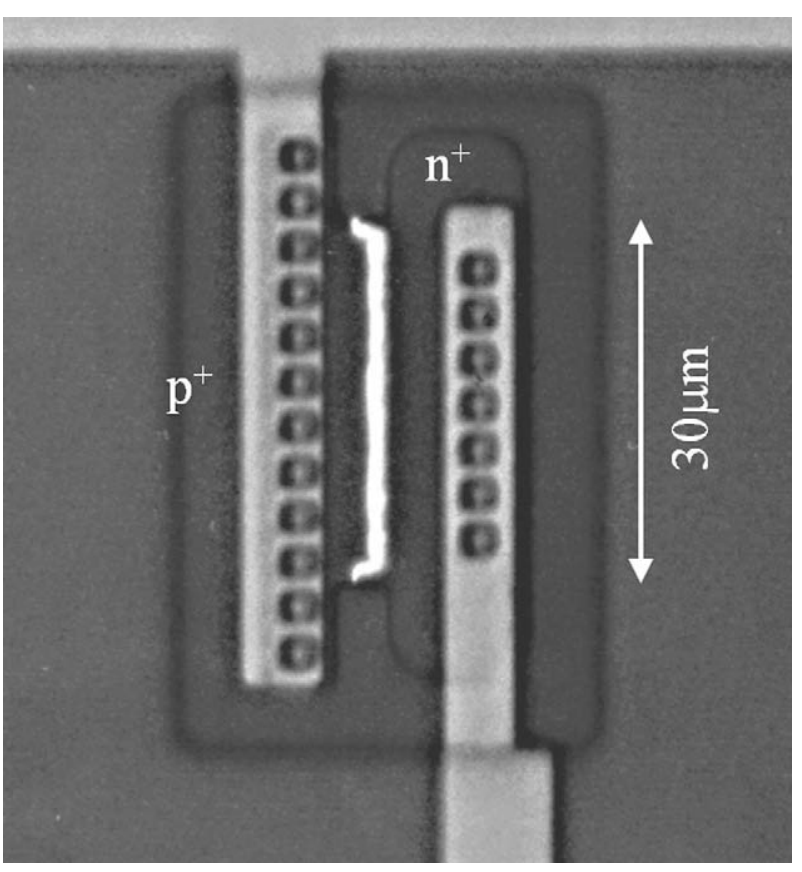

Fig. 3. Light output from the linear segment $\mathrm{n}^{+} \mathrm{p}^{+}$diode.

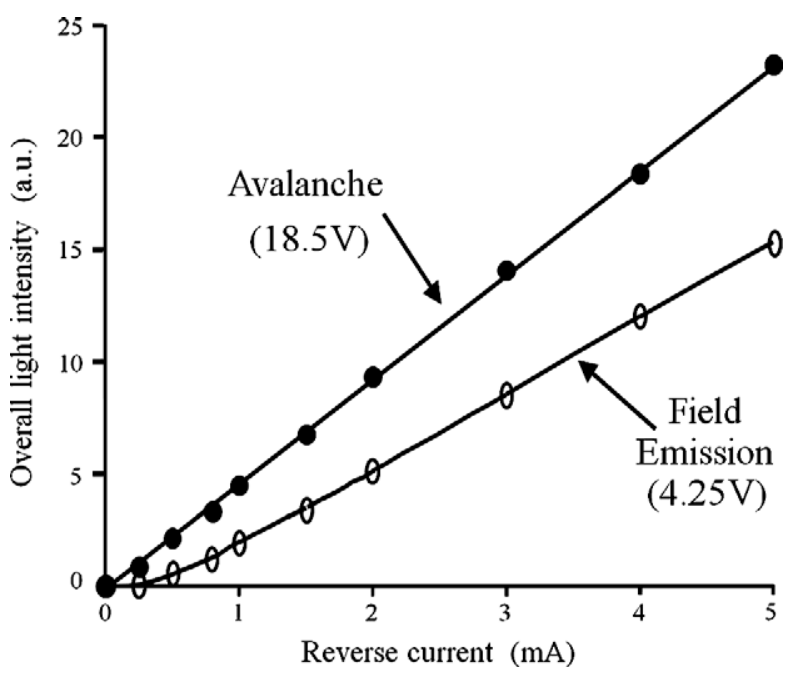

Fig. 4. Light output intensity as a function of the reverse current for low-voltage field emission $\mathrm{n}^{+} \mathrm{p}^{+}$diodes and high voltage avalanche devices [11].

\section{Extraction of the different current components}

The optical output power versus diode current curve was measured, with optical power integrated over the 400-900 nm spectrum, using a Hamamatsu R955 photomultiplier tube, and the results are shown in Fig 5. In Fig. 5 the non-linear behavior at low current levels can be observed clearly, as well as the linearity of the characteristic at larger current levels.

High voltage avalanche current devices have been observed to result in linear light emission intensity vs. reverse current characteristics [11]. Under the assumption that the tunnel current does not contribute to the light emission process, the linear avalanche current component $I_{A}$ can be shifted to the origin of the curve. Subtracting the avalanche component $I_{A}$ from the total current $I_{T O T}$ will result in the tunnel current $I_{T}$. Following this procedure, the two current components $I_{A}$ and $I_{T}$ can be plotted as a function of the

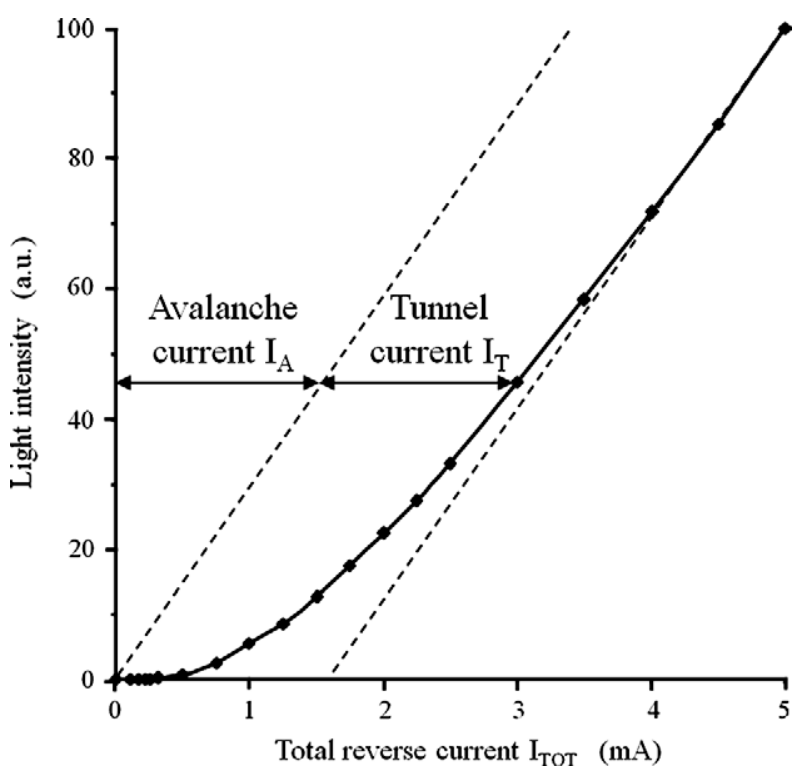

Fig. 5. Extraction of the tunnel current component $I_{T}$ from the light output intensity versus reverse current $I_{T O T}$ characteristic.

total reverse current $I_{T O T}$, as shown in Fig. 6. From this figure it follows that at low current levels the tunnel current $I_{T}$ is the dominant current flow mechanism, with almost no avalanching taking place. As the reverse current is increased the tunnel current saturates eventually and the avalanche current increases sharply to be the dominant current component mechanism at higher reverse current levels.

Using the current versus voltage relationship of Fig. 2, the two current components can also be plotted as a function of the applied reverse bias voltage, as shown in Fig. 7. From Fig. 7 it is evident that the avalanche current only starts to appear and then increases at reverse voltages of about $3.5 \mathrm{~V}$, which correlate well with previous observations using other techniques [3-6].

The avalanche gain $M_{A}$ can be calculated as the ratio of total reverse current $I_{\text {TOT }}$ to the tunnel current component $I_{T}$. At low current levels there is basically no avalanche multiplication due to impact ionization, and the avalanche gain $M_{A}$ will then be unity. As the reverse voltage increases, the depletion region of the pn junction will widen, and the probability of the tunnel current car-

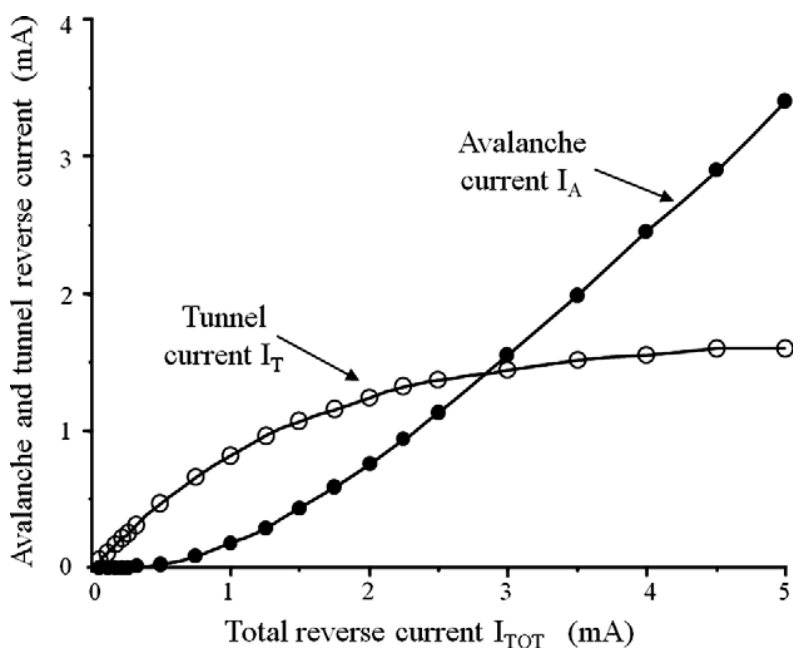

Fig. 6. The avalanche current component $I_{A}$ and field emission tunnel current component $I_{T}$ as a function of total reverse current $I_{T O T}$. 


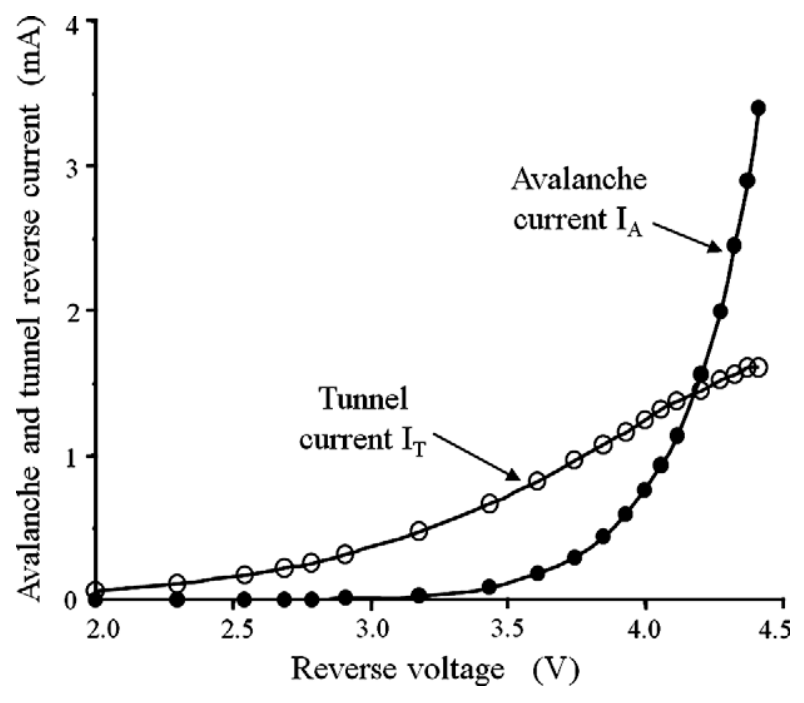

Fig. 7. The avalanche current component $I_{A}$ and field emission tunnel current component $I_{T}$ as a function of reverse bias voltage.

riers to initiate avalanche impact ionization will increase. The avalanche gain $M_{A}$ is expected to increase with an increase in reverse bias voltage. This effect is illustrated in Fig. 8. The avalanche gain measured using our light emission technique correlates very well with the avalanche gains reported by authors using different methods [3-6]. The avalanche gain shown in Fig. 8 starts to increase at reverse bias voltages in the vicinity of $3.5 \mathrm{~V}$, indicating that the impact ionization of the tunneling carriers only starts to happen at reverse bias voltages in excess of approximately $3.5 \mathrm{~V}$.

\section{Theoretical interpretation}

Our theoretical interpretation of the observed behavior is based on the theoretical models proposed earlier [3,5]. The energy band diagram of the pn junction is showed in Fig. 9 to illustrate the tunneling principle. Fig. 9 shows the tunneling of an electron $\mathrm{e}^{-}$from the valence to conduction band at the metallurgical interface of the junction where the electric field is at its highest.

In Fig. 9 the following symbols are used to illustrate the tunneling and avalanche processes:

$E_{g}$, energy band gap of silicon; $E_{l}$, energy losses due to inelastic phonon scattering; $E_{i}$, impact ionization threshold energy of elec-

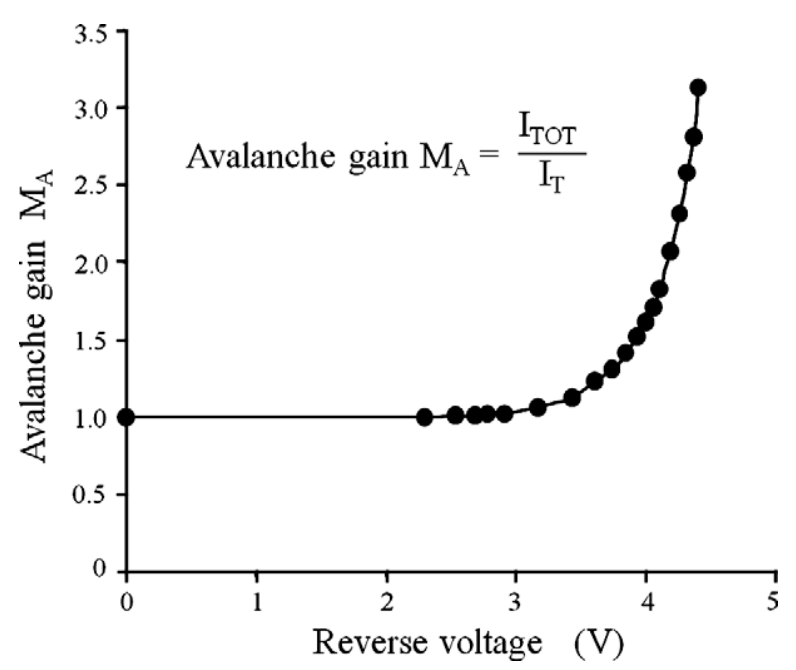

Fig. 8. The avalanche gain $M_{A}=I_{\text {ТОT }} I_{T}$ as a function of reverse bias voltage.

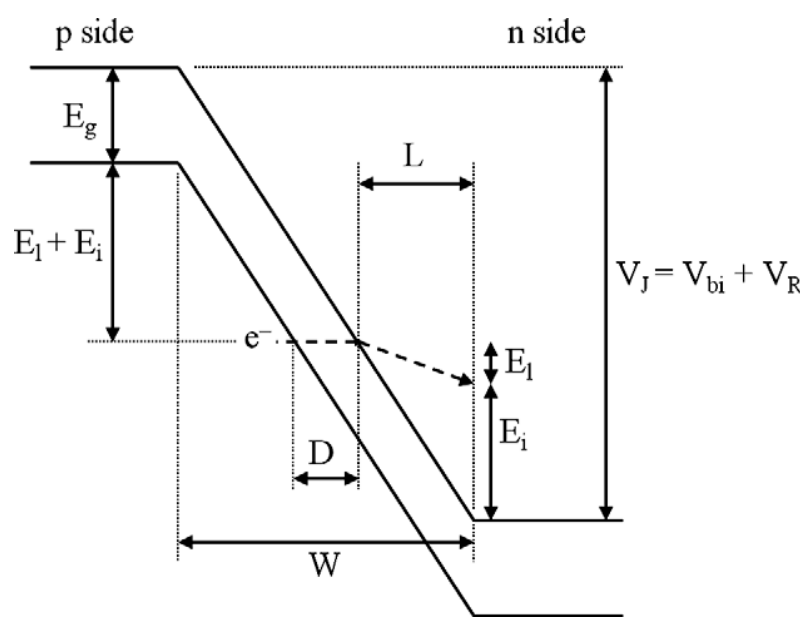

Fig. 9. The energy band diagram of the pn junction illustrating an electron tunneling from the valence to the conduction band at the position where the electric field is a maximum.

trons; $D$, barrier distance electrons tunnel through; $W$, total depletion region width; $L$, distance electrons will drift in the depletion region; $V_{J}$, total voltage across the pn junction; $V_{b i}$, built-in potential of the pn junction; $V_{R}$, applied reverse bias voltage.

An electron tunneling through the barrier width $D$ of the junction will be accelerated over a distance $L$ in the depletion region. During this acceleration it will lose energy $E_{l}$ due to inelastic phonon scattering. If the energy of the carrier before leaving the depletion region is equal to the impact ionization threshold energy $E_{i}$, there is a probability that the carrier may produce an electron-hole pair through impact ionization, and contribute to the avalanche multiplication.

Assuming the mean free path for optical phonon scattering to be $7 \mathrm{~nm}$ and the energy loss to be $0.063 \mathrm{eV}$ per collision [5], the total energy loss $E_{l}$ will be given by

$E_{l}=0.063 \times \frac{L}{7} \mathrm{eV} \quad$ with $[L]=[\mathrm{nm}]$

From Fig. 9 it is evident that the average junction potential $V_{J}$ required for at least one instance of electron-hole pair production (with $L=7 \mathrm{~nm}$ ) by a field emitted carrier is given by

$V_{J}=E_{g}+2\left(E_{i}+E_{l}\right)=1.1+2(1.8+0.063) \approx 4.8 \mathrm{~V}$

with $E_{i}=1.8 \mathrm{eV}$ [5]. In our case the built-in potential for the $\mathrm{n}^{+} \mathrm{p}^{+}$ junction is estimated as $V_{b i}=1.1 \mathrm{~V}$, giving an applied reverse bias voltage of approximately $V_{R}=3.7 \mathrm{~V}$ needed to initiate avalanche multiplication. This value agrees well with the experimental results of Figs. 7 and 8.

\section{Comparison with dynamic impedance technique}

As was noted previously, the dynamic impedance technique [4] can be used to investigate the field emission to avalanche transition region. By plotting the dynamic impedance $r_{d}=\Delta V \mid \Delta I_{T O T}$ as a function of total current $I_{T O T}$, the transition region can be observed. This measurement was performed and the experimental $r_{d}$ versus $I_{T O T}$ characteristic is shown in Fig. 10 as the solid curve. At low current levels, the tunnel (or zener) effect will be dominant, and the tunnel impedance $r_{T} \approx r_{d}$ and the tunnel current $I_{T} \approx I_{T O T}$.

The dependence of $r_{T}$ on the current $I_{T}$ in the zener mode of operation can be modeled by the simple equation [4]

$r_{T}=C_{T} I_{T}^{m_{F}}$

with the parameter $m_{F}$ the slope of the linear relationship between $\log \left(r_{T}\right)$ and $\log \left(I_{T}\right)$. At low current levels the above relationship is 


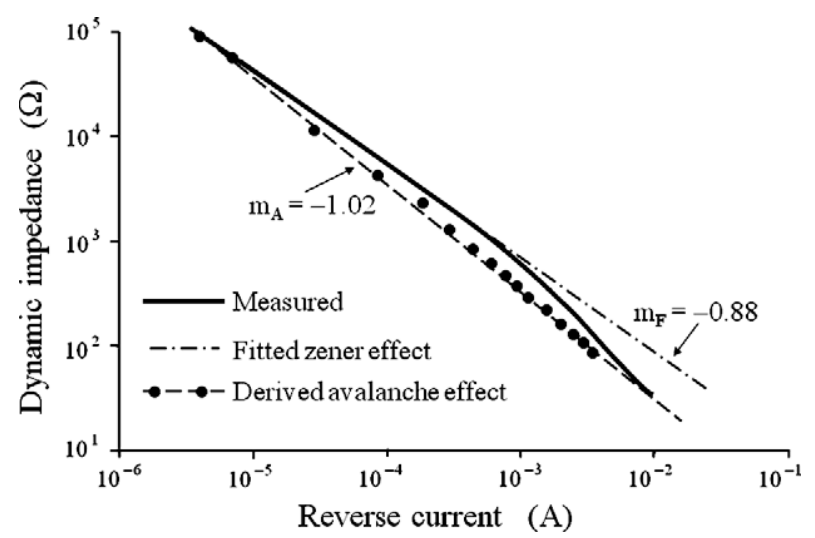

Fig. 10. The dynamic impedances as a function of reverse current.

experimentally observed. In Fig. 10 the slope $m_{F}=-0.88$ was fitted to the measured tunnel current part of the characteristic. This is in close agreement with previously reported results [4] where $m_{F}=-0.90$ was determined experimentally. The constant $C_{T}=1.59$ was the other parameter fitting the measured curve of Fig. 10.

To verify the validity of the avalanche and tunnel currents extracted, the dynamic impedance $r_{T}$ was determined for each experimental extracted value of tunnel current $I_{T}$ shown in Fig. 6. This was done using (3) with $C_{T}=1.59$ and slope $m_{F}=-0.88$. The extrapolated tunnel effect resistance values are indicated in Fig. 10 for higher current levels. If the dynamic impedances of the avalanche and tunnel effects are assumed to be effectively in parallel [4], then the avalanche dynamic impedance $r_{A}$ can be calculated from

$r_{A}=\frac{r_{T} r_{d}}{r_{T}-r_{d}}$

For example, the data point $I_{T O T}=3 \mathrm{~mA}$ is chosen in Fig. 6, with the extracted tunnel current $I_{T}=1.45 \mathrm{~mA}$ and avalanche current $I_{A}=1.55 \mathrm{~mA}$. From (3) the corresponding tunnel effect resistance $r_{T}=511 \Omega$ is calculated. The measured dynamic impedance at $I_{\text {TOT }}=3 \mathrm{~mA}$ was $r_{d}=155 \Omega$. The avalanche resistance can then be determined from (4) as $r_{A}=222 \Omega$ at the avalanche current $I_{A}=1.55 \mathrm{~mA}$. Plotting the values of $r_{A}$ versus $I_{A}$ for each data point, the derived avalanche effect graph as depicted in Fig. 10 results.

The dependence of $r_{A}$ on the current $I_{A}$ in the avalanche mode of operation can also be modeled by [4]

$r_{A}=C_{A} I_{A}^{m_{A}}$

It was found that for alloyed diodes [4] the slope $m_{A}=-0.54$, but for diffused junctions $[4,15]$ the slope should be $m_{A}=-1$. The experimentally derived avalanche effect values were fitted to (5), and the fit parameters were $m_{A}=-1.02$ and $C_{A}=0.29$. The avalanche data points and fitted graph are shown in Fig. 10. The derived value of $m_{A}=-1.02$ is very close to -1 , indicating that the avalanche current that was extracted from the light emission characteristics did exhibit typical avalanche behavior. As Fig. 10 indicates, at lower current levels the tunnel effect dominates up to about $1 \mathrm{~mA}$ total current, then a transition region is observed from $1 \mathrm{~mA}$ to $10 \mathrm{~mA}$, and above $10 \mathrm{~mA}$ the avalanche effect dominates.

\section{Theoretical evaluation of tunnel current component}

The tunnel current in a low-voltage pn junction is often modeled [16-19] by the following equation:

$I_{T}=c q A_{D} V_{R} F_{M}^{\sigma} \exp \left(\frac{-F_{0}}{F_{M}}\right)$ with $c$, tunneling prefactor which depends on direct or indirect tunneling; $A_{D}$, diode junction area; $V_{R}$, applied reverse bias voltage; $F_{M}$, maximum electric field in junction; $\sigma$, a numerical constant with a value of 1 for direct tunneling and value of 1.5 for indirect tunneling; $F_{0}$, material constant which depends on the effective mass $m^{*}$ and the bandgap $E_{g}$ of the semiconductor.

Several low-voltage silicon diodes were analyzed [17,19] and the parameter values of $F_{0}=1.9 \times 10^{7} \mathrm{~V} / \mathrm{cm}, \sigma=1.5$, and $c=1.6 \times$ $10^{14} \mathrm{~s}^{-1} \mathrm{~cm}^{-1 / 2} \mathrm{~V}^{-5 / 2}$ were fitted to the experimental results. In order to verify that the tunnel current we extracted using the light emission characteristics is due to the tunnel effect, we applied the model of (6) to the experimental data of Fig. 7. The highly doped pn junction can be modeled as a linear junction $[16,17,19]$. In the linear junction approximation it is assumed that the net doping density $N=N_{D}-N_{A}$ within the depletion region varies linearly with distance $x$ with a gradient of $\alpha=d N / d x$. The maximum electric field $F_{M}$ can be determined as a function of applied reverse bias voltage $V_{R}$ using the well-known linear junction relationship:

$F_{M}=\left[\frac{12 \varepsilon_{0} \varepsilon_{\mathrm{Si}}\left(V_{b i}+V_{R}\right)}{q \alpha}\right]^{1 / 3}$

The built-in potential for heavily doped junctions was estimated as $V_{b i}=1.1 \mathrm{~V}$. Using (6) and (7) with the constants $F_{O}=1.9 \times 10^{7} \mathrm{~V} / \mathrm{cm}$, $\sigma=1.5$, and $c=1.6 \times 10^{14} \mathrm{~s}^{-1} \mathrm{~cm}^{-1 / 2} \mathrm{~V}^{-5 / 2}$, the value of $\alpha$ was determined that gave the best fit to the experimental tunnel current of Fig. 7. The diode junction area $A_{D}$ was taken as $A_{D}=30 \mu \mathrm{m} \times 0.4 \mu \mathrm{m}$ (see Figs. 1 and 3 ). The best fit, as shown in Fig. 11, was obtained with gradient $\alpha=6.9 \times 10^{25} \mathrm{~cm}^{-4}$. From Fig. 11 it is evident that the linear junction approximation is quite accurate. Higher values of $c$ and smaller values of $F_{0}$ will result in lower values of doping gradient $\alpha$.

Although the fit parameter $\alpha=6.9 \times 10^{25} \mathrm{~cm}^{-4}$ seems a bit high, it can be reached in practice. The device structure depicted in Fig. 1 shows two overlapping heavily doped $\mathrm{n}^{+}$and $\mathrm{p}^{+}$regions. The doping profiles can be approximated by the Gaussian doping profile

$N(x)=N_{S} \exp \left(-k x^{2}\right)$

The $\mathrm{p}^{+}$region was approximated by $N_{A S}=4 \times 10^{20} \mathrm{~cm}^{-3}$ and $k_{A}=$ $6 \times 10^{9} \mathrm{~cm}^{-2}$. This resulted in a diffusion depth of about $0.4 \mu \mathrm{m}$ into the p-well. From sheet resistance values specified by the manufacturer (43 ohm/square), the $\mathrm{p}^{+}$diffusion should have an average

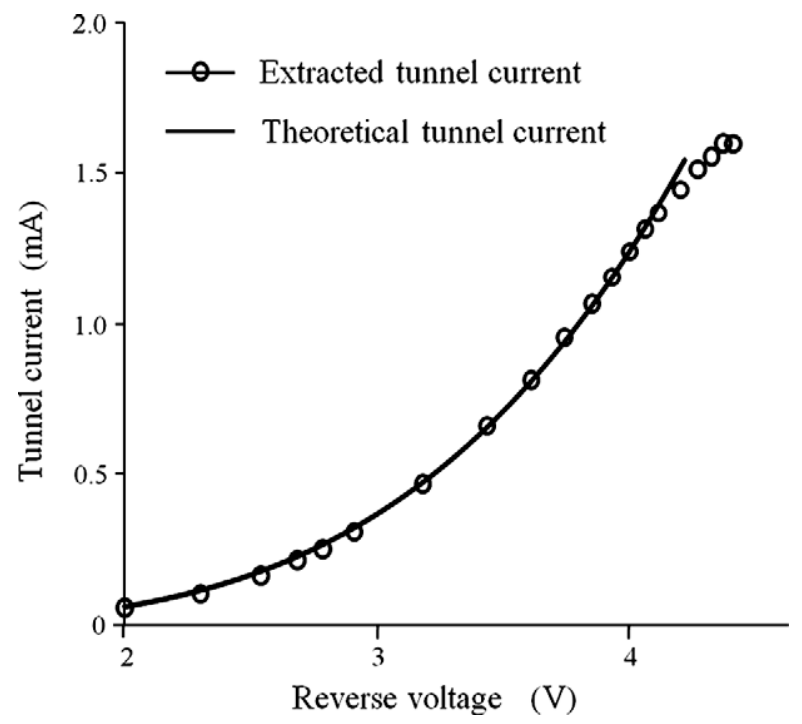

Fig. 11. The theoretical and experimentally extracted tunnel currents. 


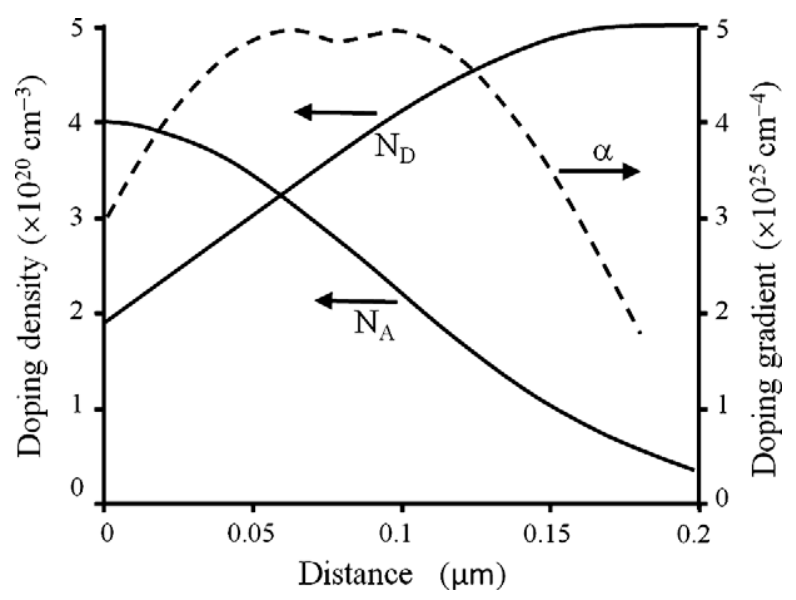

Fig. 12. Typical doping profiles and linear junction approximation for $\mathrm{n}^{+} \mathrm{p}^{+}$diodes.

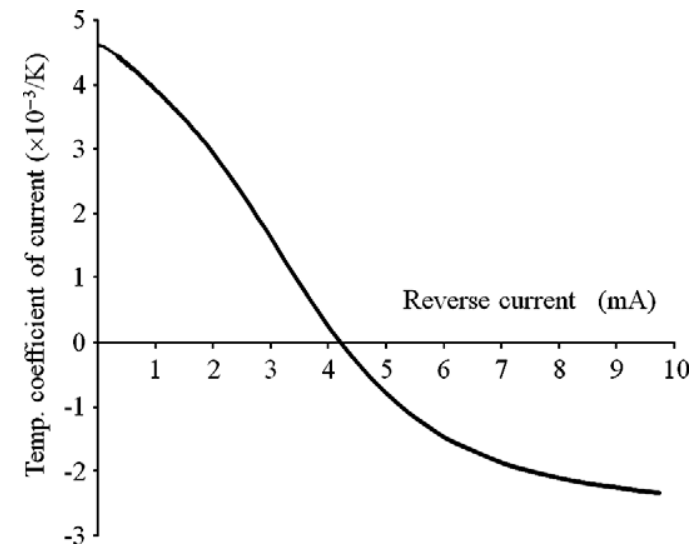

Fig. 13. The experimental temperature coefficient of the reverse current.

acceptor doping density of about $2 \times 10^{20} \mathrm{~cm}^{-3}$. The $\mathrm{n}^{+}$region was approximated by $N_{D S}=5 \times 10^{20} \mathrm{~cm}^{-3}$ and $k_{D}=3 \times 10^{9} \mathrm{~cm}^{-2}$. In Fig. 12 the two overlapping diffusions are shown on a linear scale. On the same graph $\alpha=d N(x) / d x$, with $N(\mathrm{x})=N_{D}(x)-N_{A}(x)$, is also shown. The maximum slope $\alpha$ near the metallurgical junction is comparable to the extracted value of $\alpha=6.9 \times 10^{25} \mathrm{~cm}^{-4}$.

\section{Temperature coefficient of breakdown current}

The tunneling and avalanche nature of the measured current can also be verified by determining the temperature coefficient of the reverse current. The temperature coefficient TCI of the total reverse current $I_{T O T}$ was determined by measuring the reverse current characteristics at different temperatures using the semiconductor parameter analyzer. The TCI was then calculated from

$T C I=\frac{1}{I_{T O T}} \frac{d I_{T O T}}{d T}$

The measured reverse current temperature coefficient is shown in Fig. 13. The observed positive $T C I$ at lower current levels is typical of the tunneling process, due to the bandgap temperature dependence of $F_{0} \sim E_{g}^{3 / 2}$ [17]. At higher current levels the avalanche effect will dominate with a negative $T C I$.

\section{Conclusions}

A new optical emission technique has been described to characterize the low-voltage $\mathrm{n}^{+} \mathrm{p}^{+}$silicon diode breakdown region of operation. The tunnel and avalanche components of the reverse breakdown current could be determined from the light intensity vs. reverse current characteristic. It was shown that at voltages less than $3.5 \mathrm{~V}$ the tunnel current dominates, while at larger values of reverse voltage the avalanche multiplication of the tunnel current will dominate. It has been shown theoretically that the threshold voltage for impact ionization of the tunnel-injected carriers is in the order of $3.7 \mathrm{~V}$, which correlates well with the experimental observation.

\section{Acknowledgement}

The authors thank InSiAva (Pty) Ltd. for financially supporting the research.

\section{References}

[1] Maes W, de Meyer K, van Overstraeten R. Impact ionization in silicon: a review and update. Solid-State Electron 1990;33(6):705-18.

[2] Lee CI, Ngo VH, Pan DS. New phenomena of mixed breakdown in silicon. Phys Stat Sol (b) 2006;243(4):R25-7.

[3] Chynoweth AG, MacKay KG. Internal field emission in silicon p-n junctions. Phys Rev 1957;106(3):418-27.

[4] Wienerth H. Silicon diode breakdown in the transition range between avalanche effect and field emission. Solid-State Electron 1967;10(11): 1053-62.

[5] Tyagi MS. Zener and avalanche breakdown in silicon alloyed $\mathrm{p}-\mathrm{n}$ junctions - I. Analysis of reverse characteristics. Solid-State Electron 1968;11(1):99-115.

[6] Lukaszek WA, Van der Ziel A, Chenette ER. Investigation of the transition from tunnelling to impact ionization multiplication in silicon $\mathrm{p}-\mathrm{n}$ junctions. SolidState Electron 1976;19(1):57-71.

[7] Eta ME, Haddad GI. Mixed tunneling and avalanche mechanisms in p-n junctions and their effects on microwave transit-time devices. IEEE Trans Electron Dev 1978;25(6):694-702.

[8] Massey DJ, David JPR, Tan CH, Ng BK, Rees GJ, Robbins DJ, et al. Impact ionization in submicron silicon devices. J Appl Phys 2004;95(10):5931-3.

[9] Agarwal P, Goossens MJ, Zieren V, Aksen E, Slotboom JW. Impact ionization in thin silicon diodes. IEEE Electron Dev Lett 2004;25(12):807-9.

[10] Aharoni $\mathrm{H}$, du Plessis $\mathrm{M}$. Low operating voltage integrated silicon light emitting devices. IEEE J Quantum Electron 2004;40(5):557-63.

[11] du Plessis M, Aharoni H, Snyman LW. Two- and multi-terminal silicon light emitting devices in standard CMOS/BiCMOS IC technology. Phys Stat Sol (a) 2004;201(10):2225-33.

[12] Jaguiro P, Katsuba P, Lazarouk S, Smirnov A. Porous silicon avalanche LEDs and their application in optoelectronics and information displays. Acta Phys Pol A 2007;112(5):1031-6.

[13] Sze SM. Physics of semiconductor devices. New York: John Wiley \& Sons; 1979

[14] Yang ES. Fundamentals of semiconductor devices. New York: McGraw-Hill Inc.; 1978.

[15] Shockley W. Problems related to p-n junctions in silicon. Solid-State Electron 1961;2(1):35-67.

[16] Fair RB, Wivell HW. Zener and avalanche breakdown in As-implanted lowvoltage Si n-p junctions. IEEE Trans Electron Dev 1976;23(5):512-8.

[17] Hurkx GAM. On the modelling of tunnelling currents in reverse-biased p-n junctions. Solid-State Electron 1989;32(8):665-8.

[18] Liou JJ. Modeling the tunnelling current in reverse-biased $\mathrm{p} / \mathrm{n}$ junctions. SolidState Electron 1990;33(7):911-72.

[19] Hurkx GAM, de Graaff HC, Kloosterman WJ, Knuvers MPG. A new analytical diode model including tunneling and avalanche breakdown. IEEE Trans Electron Dev 1992;39(9):2090-8. 